\title{
miR-448 targets Rab2B and is pivotal in the suppression of pancreatic cancer
}

\author{
JING JIN, YINGSHENG WU, DONGKAI ZHOU, QIANG SUN and WEILIN WANG
}

\begin{abstract}
Key Laboratory of Precision Diagnosis and Treatment for Hepatobiliary and Pancreatic Tumor of Zhejiang Province, Division of Hepatobiliary and Pancreatic Surgery, The First Affiliated Hospital, School of Medicine, Zhejiang University, Hangzhou, Zhejiang 310003, P.R. China
\end{abstract}

Received March 7, 2018; Accepted July 3, 2018

DOI: $10.3892 /$ or.2018.6562

\begin{abstract}
Improvements in survival rates for pancreatic cancer have been slow and the morality rate continues to increase in patients. MicroRNA (miR)-448 is reported to be significantly downregulated in several types of cancer. In this study, Rab2B is target of miR-488 was confirmed by bioinformatics analysis and validated using a luciferase reporter assay. A total of 72 cases of pancreatic cancer in patients diagnosed at The First Affiliated Hospital, School of Medicine, Zhejiang University (Hangzhou, China) were enrolled, and cancer specimens and their adjacent normal tissues were collected for analysis. The expression levels of miR-448 and Rab2B in these tissues and in pancreatic cancer cell lines were quantified using reverse transcription-polymerase chain reaction analysis. miR-448 overexpression was achieved by cell transfection. Protein expression was assessed using western blot analysis. Cell viability, cell cycle and apoptosis were analyzed using CCK- 8 assay and flow cytometry, respectively. The results revealed a negative correlation between miR-448 and Rab2B in the pancreatic tissues and cell lines. The results of bioinformatics analysis indicated that miR-448 directly targeted Rab2B. Aberrant miR-448 levels in PANC-1 cells downregulated the expression of Rab2B, and significantly decreased cell proliferation and promoted apoptosis of cancer cells. It was also found that miR-448 mimics resulted in $\mathrm{G}_{0} / \mathrm{G}_{1}$ cell cycle arrest and affected the expression of cell cycle regulators, including cyclin D1, p21 and p27. In addition, the miR-448 mimics led to inactivation of the Akt/Mammalian target of rapamycin signaling pathway. The miR-448 mimics induced
\end{abstract}

Correspondence to: Dr Weilin Wang, Key Laboratory of Precision Diagnosis and Treatment for Hepatobiliary and Pancreatic Tumor of Zhejiang Province, Division of Hepatobiliary and Pancreatic Surgery, The First Affiliated Hospital, School of Medicine, Zhejiang University, 79 Qingchun Road, Hangzhou, Zhejiang 310003, P.R. China E-mail:wam@zju.edu.cn

Key words: microRNA-448, Rab2B, pancreatic cancer, cell proliferation, cell cycle, apoptosis apoptosis and activated the expression of caspase-3, caspase-9 and poly(ADP-ribose) polymerase. The results suggested that miR-448 was a negative regulator of Rab2B and promoted cell cycle arrest and apoptosis in pancreatic cancer.

\section{Introduction}

According to the cellular direction of the differentiation of tumor cells, malignant neoplasms of the pancreas can be classified into three categories: Ductal, acinar, and neuroendocrine (1). Pancreatic ductal adenocarcinoma, which accounts for $\sim 90 \%$ of malignant pancreatic tumors, has become the fourth leading cause of cancer-associated mortality in the USA and has an annual mortality rate of 227,000 worldwide (2). Based on a previous report, despite decades of research, advances in terms of survival rates have been slow for pancreatic cancer. The 5-year survival rate for patients diagnosed with pancreatic cancer is $8 \%$, and the morality rate continues to rise by $0.3 \%$ per year among men (3). The poor prognosis is primarily attributable to late stage disease at diagnosis and inherent cancer cell resistance to standard chemotherapy (4-6).

Due to the lack of a more sensitive and precise biomarkers under imaging detection, molecular technology has been applied to identify biomarkers that have the potential to detect disease at the early stage and assist in predicting disease progression (4). The significance of microRNA (miRNA) profiling has been highlighted in a variety of human diseases, for example, malignant tumors (7-9). miRNAs, a group of non-coding RNA molecules of 18-22 nucleotides in length, are key in cellular functions, including apoptosis, metabolism, cell proliferation and differentiation. Aberrant miRNAs levels can lead to gene mutations, epigenetic modifications or defects in the miRNA processing pathway (10).

The Rab family, known alternatively as small GTP-binding proteins, are members of the Ras-like small GTPase superfamily. It has been demonstrated that the family contributes to the control of intracellular membrane trafficking in eukaryotic cells (11). The majority of Rab proteins have been shown to be expressed in numerous tissues, although the expression levels vary among diverse tissues and cells. Tissue specificity and cell specificity have been identified in several Rab proteins $(12,13)$. Rab2 contains a conservative GTP binding domain and a variable $\mathrm{N}$-terminus domain and $\mathrm{C}$-terminus 
domain (14). As a proto-oncogene, Rab2 has been reported to be aberrantly expressed in the peripheral blood cells of solid tumors in patients, and to be gradually downregulated during the treatment process (15).

To the best of our knowledge, the association between pancreatic cancer and the dysregulation of miRNA-448 (miR-448) has not been investigated previously. In addition, the regulation of the expression of Rab2B by miRNAs remains to be fully elucidated. The present study aimed to examine the role of miR-448 in pancreatic cancer in vitro. In addition, the study intended to determine whether the experimental manipulation of miR-448 in pancreatic cancer cells can regulate the expression of Rab2B and inhibit the growth of pancreatic cancer at a molecular level.

\section{Materials and methods}

Tumor samples and cells. A total of 72 patients with pancreatic cancer admitted to the First Affiliated Hospital, School of Medicine, Zhejiang University (Hangzhou, China) were accepted into the present study. Cancer tissue specimens and adjacent normal pancreatic tissue samples were collected from surgical tumor resections from the patients. The basic clinical and pathological data of these patients were collected with their signed written informed consent. All experimental protocols were approved by the Ethics Committee of The First Affiliated Hospital, School of Medicine, Zhejiang University.

The HPDE6-C7 normal pancreatic cell line, and the AsPC-1, BxPC-3, Capan-1, CFPAC-1, HPAC, Hs 766T and PANC-1 pancreatic cancer cell lines were purchased from Nanjing Cobioer Biotechnology Co., Ltd. (Nanjing, China). The cells were cultured in Dulbecco's modified Eagle's medium (DMEM) containing 10\% fetal calf serum (FCS; Thermo Fisher Scientific, Inc., Waltham, MA, USA) with 5\% $\mathrm{CO}_{2}$ in an incubator at $37^{\circ} \mathrm{C}$. The medium was replaced once every 2 days. Cell passage cultivation was performed when the cells had grown to $90 \%$.

Cell grouping. The PANC-1 cell line was selected as the subject of investigation for the in vitro experiments. To investigate the role of miR-448 in the regulation of the expression of Rab2B in pancreatic cancer, the cells were randomly divided into three groups: The Control, the mock and the mimics group. The cells in the mimics group were transfected with miR-448 mimics. In the mock group, the cells were transfected with blank plasmids, and a normal PANC-1 cell group was set as a negative control group. The recombinant plasmids used in the present study were obtained from Beijing SyngenTech, Co., Ltd. (Beijing, China). The transfection processes were performed using Lipofectamine 2000 reagent (Thermo Fisher Scientific, Inc.), according to the manufacturer's protocol.

Bioinformatics analysis. Bioinformatics analysis was performed using prediction software. The target genes of miR-448 were predicated via miRanda (http://www. microrna.org/microrna/home.do), miRDB (http://www.mirdb. org/), PicTar (http://pictar.mdc-berlin.de/) and TargetScan (http://www.targetscan.org/vert_71/). The probable functions of the miRNA were predicted via the Database for Annotation, Visualization and Integrated Discovery (https://david.ncifcrf.gov/).

Luciferase reporter assay. The 3'-untranslated region (UTR) fragment of Rab2B with a binding site for miR-448 was cloned into luciferase vectors (Promega Corp., Madison, WI, USA). The PANC-1 cells were seeded into 96-well plates at a density of $1 \times 10^{5}$ cells/well 1 day prior to transfection. The control luciferase reporter plasmid, Rab2B 3'-UTR or mutated Rab2B 3'-UTR (GeneCopoeia, Inc., Rockville, MD, USA) was co-transfected with either miR-448 mimic or miR-448 negative control using Lipofectamine 3000 (Thermo Fisher Scientific, Inc.). At $48 \mathrm{~h}$ post-transfection, the luciferase activity was determined with the Secrete-Pair ${ }^{\mathrm{TM}}$ Dual-Luciferase Reporter assay (GeneCopoeia). The miR-448 mimics (mature sequence: UUGCAUAUGUAGGAUGUCCCAU) (miR10001532-1-5) and miR-448 inhibitors (miR20001532-1-5) are obtained from Guangzhou Ribobio Technology Co., Ltd. (Guangzhou, China).

CCK-8 assay. Cell proliferation in the control, mock and mimics group were determined using a CCK-8 assay (Shanghai Haling Biotechnology, Co., Ltd., Shanghai, China). The cells were seeded into a 96-well plate (100 $\mu \mathrm{l} /$ well) and cultured in an incubator with $5 \% \mathrm{CO}_{2}$ at $37^{\circ} \mathrm{C}$ for $4 \mathrm{~h}$. Subsequently, $10 \mu \mathrm{l}$ of CCK-8 reagent was added to the cells, which were placed into a $\mathrm{CO}_{2}$ incubator again for 1-4 h. The optical density (OD) values were read by an iMark microplate absorbance reader (Bio-Rad Laboratories, Inc., Hercules, CA, USA) at the wavelength of $450 \mathrm{~nm}$.

Flow cytometry (FCM). The cells were digested by $0.25 \%$ trypsin-EDTA (Beyotime Institute of Biotechnology, Haimen, China) and collected by centrifugation at $500 \times \mathrm{g}$ at $4^{\circ} \mathrm{C}$ for 5 min and washed three times with phosphate-buffered saline (PBS). The precipitated cells were resuspended and fixed in $70 \%$ absolute alcohol. Subsequently, for the purpose of analyzing cell cycle, the cells were washed with PBS and centrifuged at $500 \mathrm{x} \mathrm{g}$ at $4^{\circ} \mathrm{C}$ for $5 \mathrm{~min}$ to collect the precipitate. Propidium iodide (PI) was added for staining. The cell cycle status was determined using an EPICS XL-MCL FCM system (Beckman Coulter, Inc., Brea, CA, USA).

Cells in the logarithmic phase were collected and seeded into 6-well plates $\left(3 \times 10^{5} /\right.$ well $)$. The cells were then digested in EDTA-free trypsin (Shanghai Lanpai Biotechnology Co., Ltd., Shanghai, China), and stained with Annexin V-FITC and PI (Shanghai Lanpai Biotechnology Co., Ltd.). The cells were then incubated in the dark for $15 \mathrm{~min}$ at room temperature. The apoptotic rate of the cells in each group was detected using the EPICS XL-MCL FCM (Beckman Coulter, Inc.) with an excitation wavelength of $488 \mathrm{~nm}$ and emission wavelength of $530 \mathrm{~nm}$.

Determination of caspase activities. The activities of caspase-3/-9 were evaluated using caspase-3 and caspase-9 colorimetric assay kits, according to the manufacturer's protocol. The cells were lysed in lysis buffer. Substrates for caspase-3 and caspase-9 (Ac-DEVD-pNA and Ac-LEHD-pNA) were added to the cell lysates (50 $\mu \mathrm{g}$ proteins). The protein concentration was determined by BCA assay kit (Thermo Fisher Scientific, Inc.). The samples were then incubated at 
Table I. Association between Rab2B and clinical data of patients with pancreatic cancer.

\begin{tabular}{lccc}
\hline TNM cancer stage & Male/female & Age $(<45 / \geq 45$ years $)$ & Rab2B expression (low/high) \\
\hline I & $11 / 14$ & $8 / 17$ & $12 / 13$ \\
II & $18 / 11$ & $7 / 22$ & $6 / 23$ \\
III & $12 / 6$ & $3 / 15$ & $3 / 15$ \\
P-value & 0.258 & 0.514 & $0.036^{\mathrm{a}}$ \\
\hline
\end{tabular}

${ }^{\mathrm{a}} \mathrm{P}<0.05$, determined by $\chi^{2}$ test. TNM, tumor-node-metastasis.

$37^{\circ} \mathrm{C}$ for $1 \mathrm{~h}$. The absorbance was measured at $405 \mathrm{~nm}$ using a microplate reader (Bio-Rad Laboratories).

Reverse transcription-quantitative polymerase chain reaction $(R T-q P C R)$ analysis. Quantification of the expression of miR-448 was determined using a TaqMan miRNA assay (Thermo Fisher Scientific, Inc.). Reverse transcription of $10 \mathrm{ng}$ of template RNA was performed using the TaqMan MicroRNA Reverse Transcription kit and miRNA-specific stem-loop primers. The expression was normalized by U6. The primers used were as follows: miR-448, forward, 5'-TTG CATATGTAGGATGTCCCAT-3' and reverse, 5'-CTCAAC TGGTGTCGTGGAGTCGGCAATTCAGTTGAGATGGGA CA-3'; and U6, forward, 5'-ACGAATTGCGTGTCATCCT-3' and reverse, 5'-ACGAATTTGCGTGTCATCCT-3'.

To determine the mRNA levels of other genes, including Rab2B, total RNA (2 $\mu \mathrm{g})$ was first reverse transcribed using the Takara PrimeScript RT reagent kit (Takara Bio, Inc., Otsu, Japan) (containing PrimeScript buffer and PrimeScript $^{\mathrm{TM}}$ RT Enzyme). Quantification of mRNA was performed with the TaqMan Gene Expression Assay (Thermo Fisher Scientific, Inc.). The thermocycling conditions were as follows: $5 \mathrm{~min}$ pretreatment at $94^{\circ} \mathrm{C} ; 94^{\circ} \mathrm{C}$ for $10 \mathrm{sec}, 60^{\circ} \mathrm{C}$ for $30 \mathrm{sec}\left(30\right.$ cycles); final extension at $72^{\circ} \mathrm{C}$ for $10 \mathrm{~min}$. Target gene expression was normalized on the basis of glyceraldehyde-3-phosphate dehydrogenase (GAPDH). The quantification was determined by the $2^{-\Delta \Delta \mathrm{Cq}}$ method. The primer sequences for the gene were as follows: Rab2B, forward, 5'-GGTCCGGGAAGTCCATACTC-3' and reverse, 5'-GGCTGGAACCGCTTATCTGT-3'; and GAPDH, forward, 5'-AAGCCTGCCGGTGACTAAC-3' and reverse, 5'-GCA TCACCCGGAGGAGAAAT-3'.

Western blot analysis. The total protein of the cells in each group was extracted using a ProteoPrep ${ }^{\circledR}$ Total Extraction Sample kit (Sigma-Aldrich; EMD Millipore, Billerica, MA, USA). The cell lysates were prepared in cell lysis buffer, and the supernatant was collected following centrifugation at $500 \mathrm{x} \mathrm{g}$ at $4^{\circ} \mathrm{C}$ for $10 \mathrm{~min}$. The concentration of the protein was determined using a Bradford assay (Bio-Rad Laboratories) and $30 \mu \mathrm{g}$ of each protein sample was separated via 10-15\% SDS-PAGE (Merck Millipore, Darmstadt, Germany). The membranes were blocked with 5\% skimmed milk for $2 \mathrm{~h}$ at room temperature. Then, the membranes were incubated with primary antibodies at $4^{\circ} \mathrm{C}$ overnight. The membranes were subsequently incubated with a horseradish peroxidase-conjugated secondary antibody (dilution 1:2,000; cat. no. ab205718; Abcam) at room temperature for $1 \mathrm{~h}$. The primary antibodies were as follows: Anti-Rab2B (dilution 1:1,000; cat. no. ab95952; Abcam); anti-cyclin D1 (dilution 1:10,000; cat. no. ab134175; Abcam); anti-cyclin-dependent kinase inhibitor 1B (p27; dilution 1:5,000; cat. no. ab32034; Abcam); anti-procaspase-9 (dilution 1:500; cat. no. ab135544; Abcam); anti-procaspase-3 (dilution 1:1,000; cat. no. ab32150; Abcam); anti-p-Akt (dilution 1:500; cat. no. ab38449; Abcam); anti-p21 (dilution 1:1,000; cat. no. ab109520; Abcam); anti-Akt (dilution 1:6,000; cat. no. ab81283; Abcam); anti-poly(ADP-ribose) polymerase (PARP; dilution 1:5,000; cat. no. ab32138; Abcam); anti-GAPDH (dilution 1:2,500; cat. no. ab9485; Abcam). Blots were developed with enhanced chemiluminescence detection kit (GE Healthcare, Chicago, IL, USA). The density of the blots was read with Quantity One software version 4.6.2 (Bio-Rad Laboratories).

Statistical analysis. Statistical analyses were performed using SPSS software, version 22.0 (IBM SPSS, Armonk, NY USA) and GraphPad Prism software 6.0 (GraphPad Software, Inc., La Jolla, CA, USA). Each experiment was repeated three times. Data are presented as the mean \pm standard deviation. Student's t-test and one-way analysis of variance (ANOVA) with Turkey's test were performed to calculate statistical significance. Spearman's rank order was used to analyze the correlations between variables. Kaplan-Meier survival curve analysis was used to show the survival rates. $\mathrm{P}<0.05$ was considered to indicate a statistically significant difference.

\section{Results}

Levels of Rab2B are negatively correlated with the expression of miR-488 in pancreatic cancer tissues and cell lines. A total of 72 patients with pancreatic cancer with an average age of $55.28 \pm 4.08$ years were accepted into the present study (Table I). The mRNA levels of miR-448 and Rab2B in pancreatic cancer samples and their adjacent normal tissues were examined using RT-qPCR analysis. In the cancer tissues, the expression of miR-448 was inhibited, whereas the level of Rab2B was significantly enhanced ( $\mathrm{P}<0.05$; Fig. 1A and $\mathrm{B})$. In addition, a significant negative correlation between the expression of miR-448 and Rab2B was detected ( $\mathrm{P}<0.01$; Fig. 1C). The patients were then divided either into a higher Rab2B group and lower Rab2B group based on the median value of the expression of Rab2B. Survival analysis revealed that 

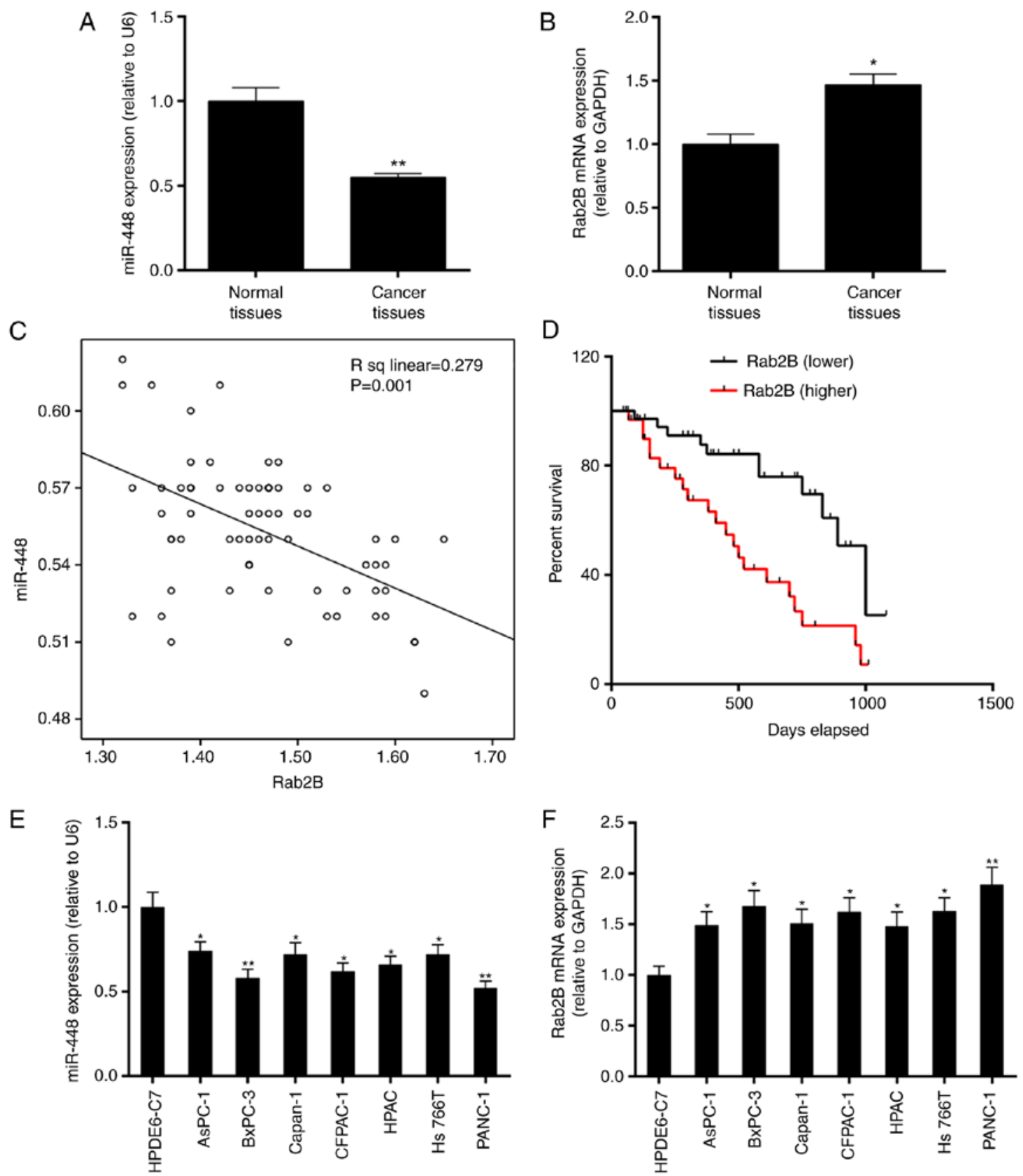

Figure 1. Levels of miR-448 and Rab2B in pancreatic tissues and cell lines (HPDE6-C7, AsPC-1, BxPC-3, Capan-1, CFPAC-1, HPAC, Hs 776T and PANC-1). (A) Expression of miR-448 was silenced in pancreatic cancer tissues. (B) Expression of Rab2B was high in pancreatic cancer tissues. (C) Linear correlation of mRNA levels between miR-448 and Rab2B. (D) Survival analysis showed the survival rate of patients with pancreatic cancer with a higher level of Rab2B was lower than that of patients with a lower expression level of Rab2B. (E) Levels of miR-448 in pancreatic cancer cell lines were significantly lower than in normal cells (HPDE6-C7). (F) mRNA levels of Rab2B in pancreatic cancer cell lines were higher compared with that in normal cells (HPDE6-C7). Data are shown as the mean \pm standard deviation. ${ }^{*} \mathrm{P}<0.05,{ }^{* *} \mathrm{P}<0.01$, vs. normal tissues or cells. miR, microRNA.

patients with a weaker expression of Rab2B were likely to survive longer than those who had a high expression level of Rab2b (P<0.01; Fig. 1D).

Through the analysis of normal and cancer cell lines of the pancreas (HPDE6-C7, AsPC-1, BxPC-3, Capan-1, CFPAC-1, HPAC, Hs 776T and PANC-1), lower levels of miR-448 and higher levels of Rab2B were detected in the cancer cell lines $(\mathrm{P}<0.05$; Fig. 1E and $\mathrm{F})$.

Identification of Rab2B as the direct target of miR-448. The in silico analysis of human miR-448 started with surveys of its target-prediction. The four prediction software packages,
miRanda, miRDB, PicTar and TargetScan, respectively identified 3,488, 483, 1,127 and 684 target genes of human miR-448. On the basis of these predicted target genes, a total of 104 target genes of intersection of miR-448 were isolated by the means of a Venn diagram (Fig. 2A). Subsequently, 20 annotations of biological processes in association with miR-448 were predicted using Gene Ontology analysis $(\mathrm{P}<0.01$; Fig. 2B). The results revealed that the genes targeted by miR-448 were primarily abundant in the cytoplasm and plasma membrane, and mainly functioned in protein binding. Subsequent analysis of the alignment of miR-448/Rab2B was performed to confirm the target association between miR-448 and Rab2B. 

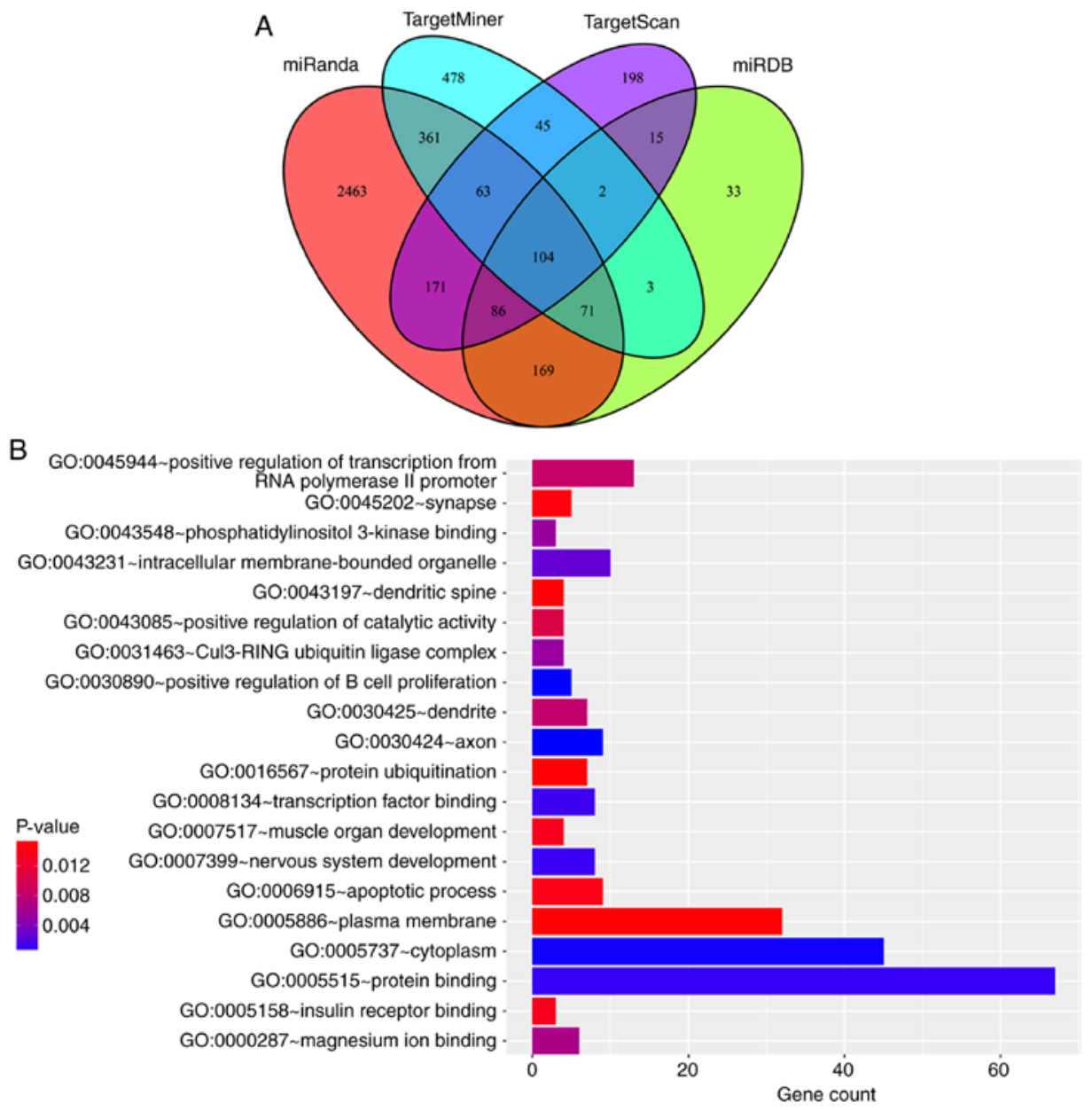

Figure 2. Target of miR-448 identified by bioinformatics analysis and luciferase reporter analysis. (A) A total of 90 reliable target genes of miR-448 were identified through intersection calculation of predicted target genes by miRanda, miRDB, PicTar and TargetScan. (B) GO analysis of gene function found 20 annotations of miR-448-associated biological process, which were mainly enriched in processes of regulation of gene expression, cell proliferation and signal transduction. miR, microRNA; GO, Gene Ontology.

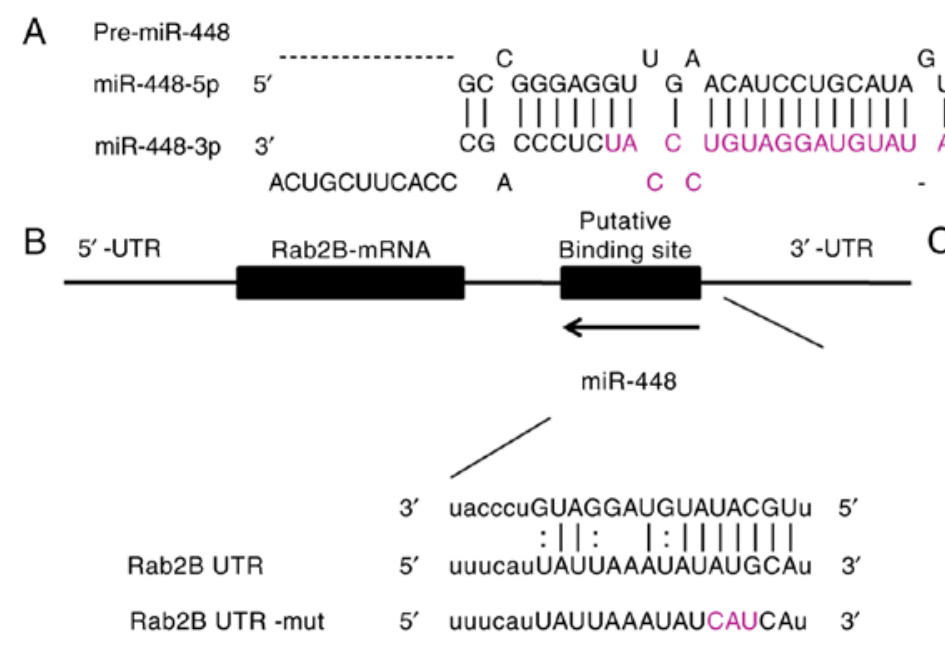


A

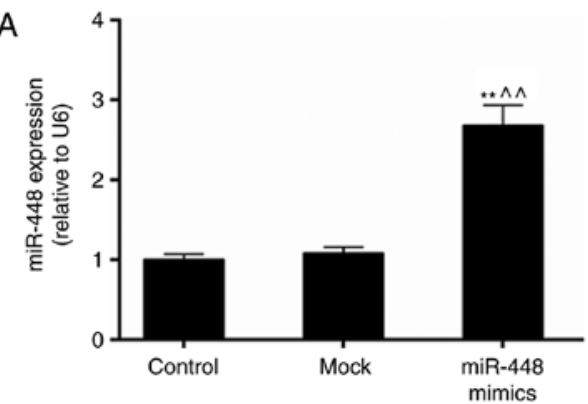

C

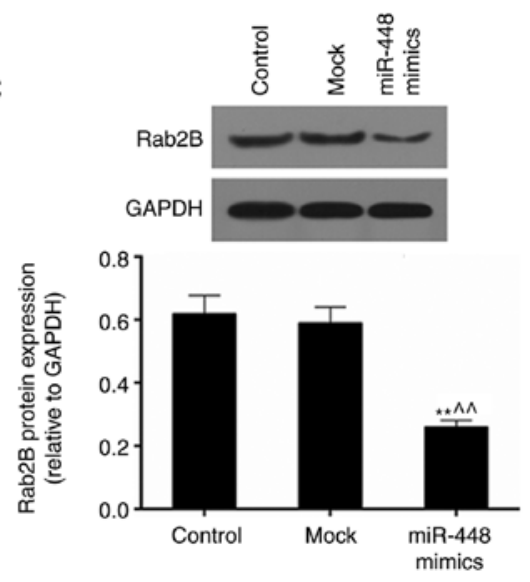

B

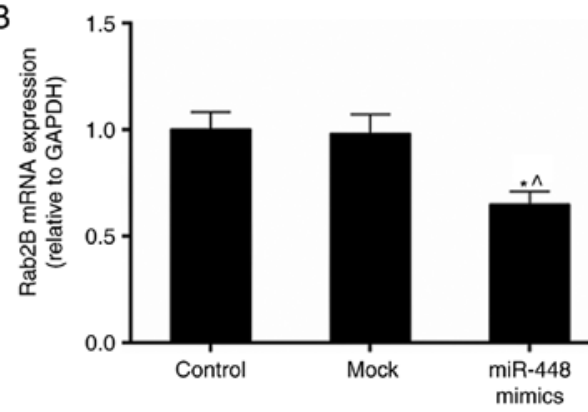

D

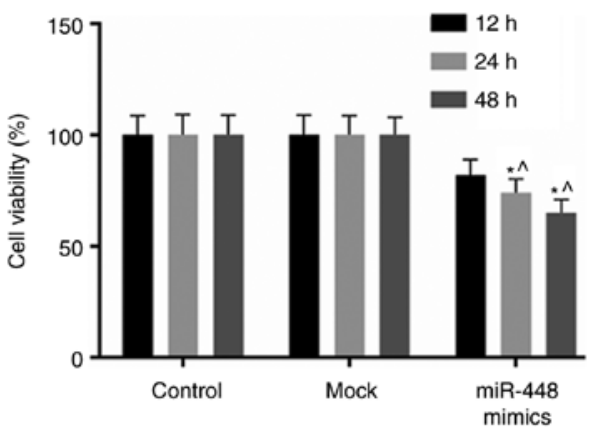

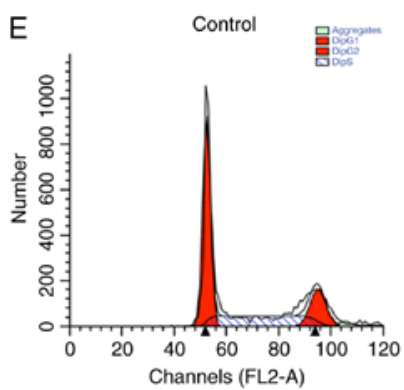
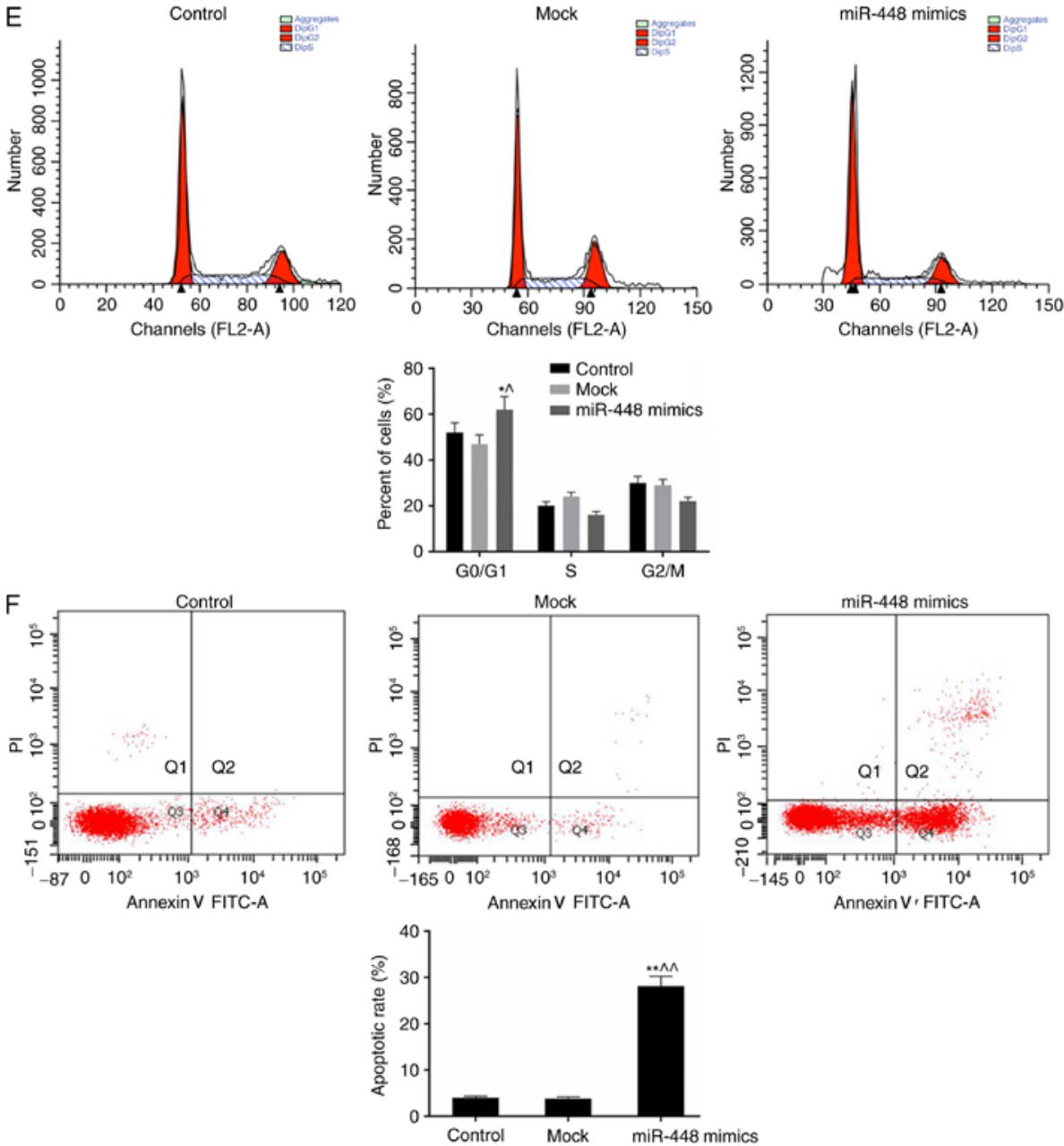

Figure 4. Overexpression of miR-448 affects cell proliferation, migration and invasion. (A) Ectopic expression of miR-448 was confirmed, which (B) decreased the mRNA level of Rab2B. (C) Protein expression of Rab2B was inhibited by miR-448 mimics. (D) Overexpression of miR-448 decreased the proliferative ability of PANC-1 cells. (E) miR-448 induced $\mathrm{G}_{0} / \mathrm{G}_{1}$ cell cycle arrest in PANC-1 cells. (F) miR-448 promoted the apoptotic rate of PANC-1 cells. Data are shown as the mean \pm standard deviation of three independent experiments. ${ }^{*} \mathrm{P}<0.05,{ }^{* *} \mathrm{P}<0.01$, vs. control; ${ }^{\wedge} \mathrm{P}<0.05,{ }^{\wedge} \mathrm{P}<0.01$, vs. mock. miR, microRNA; glyceraldehyde-3-phosphate dehydrogenase. 
A
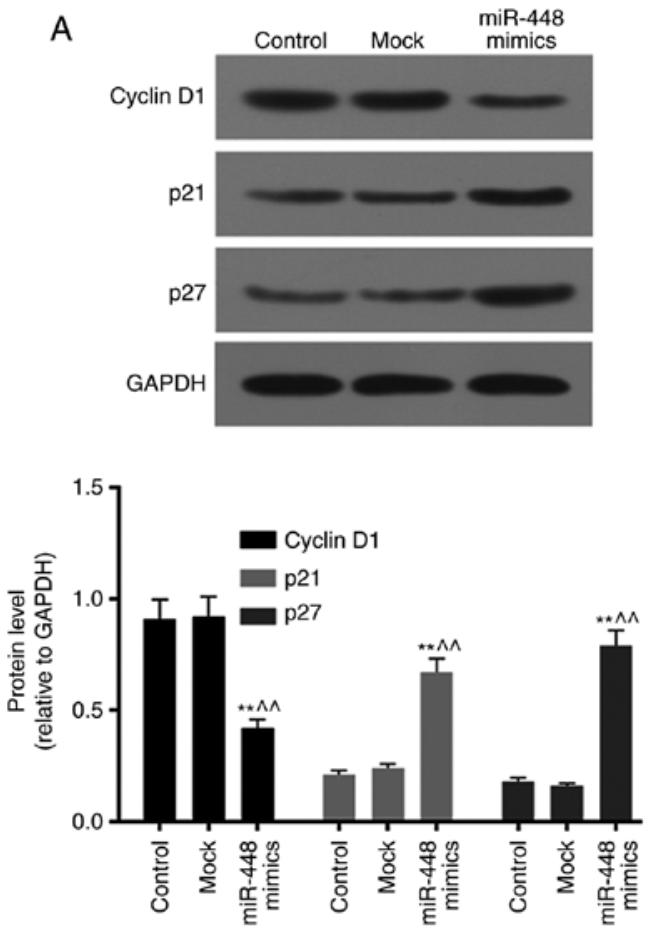

C

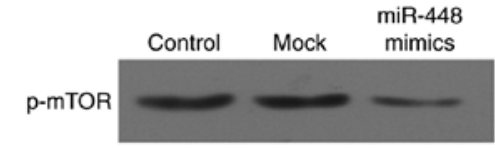

mTOR

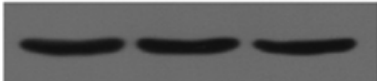

GAPDH
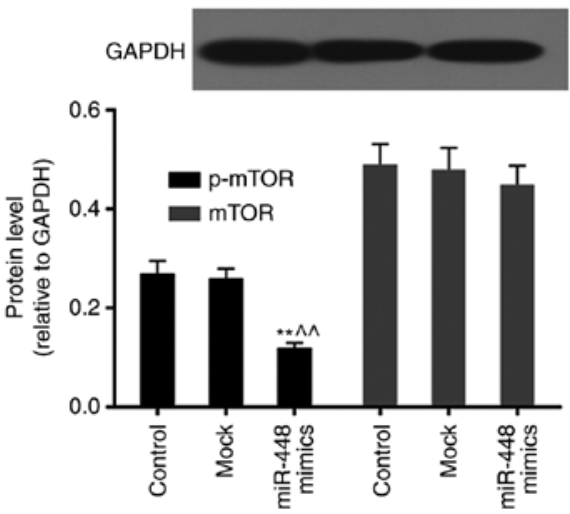

B
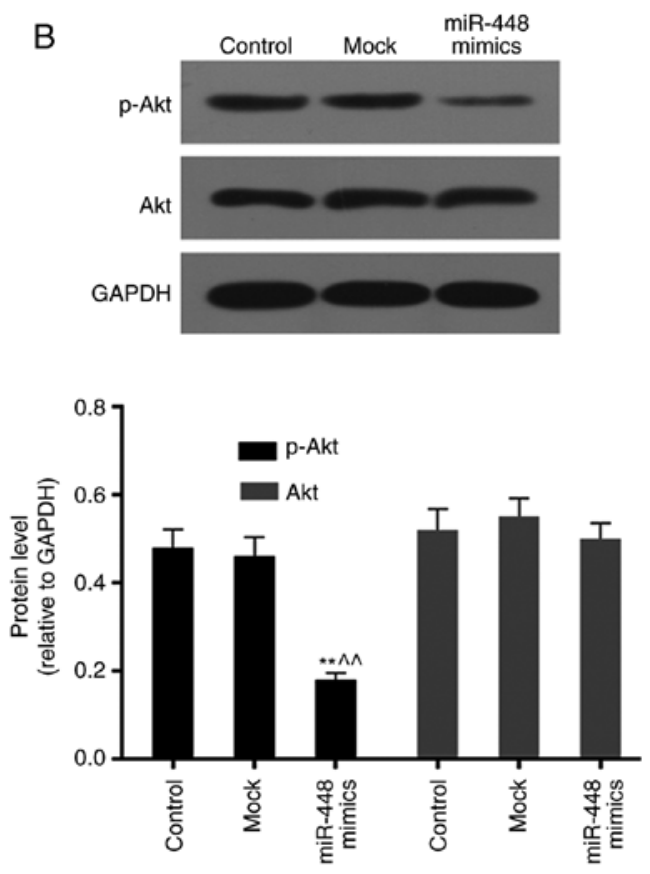

D
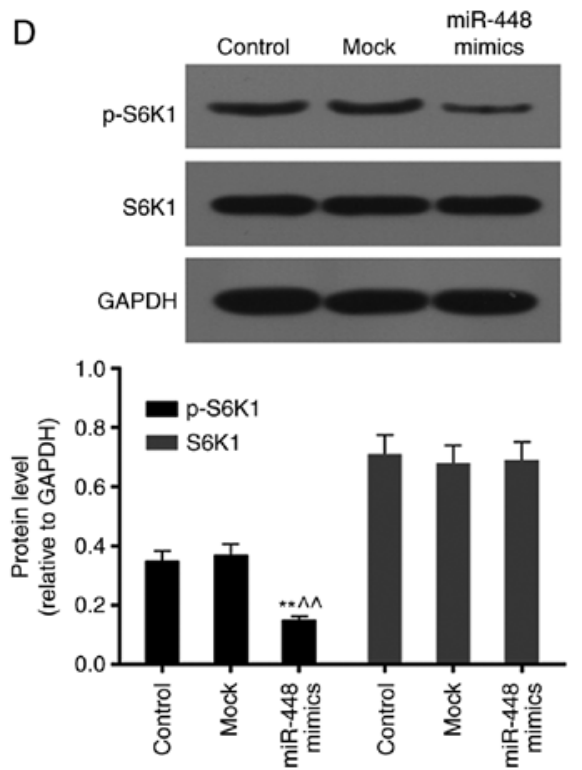

Figure 5. miR-448 mimics affect the expression of cell cycle regulators and inhibit the Akt/mTOR/S6K1 pathway. (A) Ectopic expression of miR-448 inhibited the expression of Cyclin D1 but upregulated the expression of p21 and p27. The phosphorylation of (B) Akt, (C) mTOR and (D) S6K1 was suppressed by miR-448 mimics. Data are shown as the mean \pm standard deviation of three independent experiments. ${ }^{* * *} \mathrm{P}<0.01$, vs. control; ${ }^{\wedge} \mathrm{P}<0.01$, vs. mock. miR microRNA; mTOR, Mammalian target of rapamycin; p-, phosphorylated.

each group was determined $24 \mathrm{~h}$ following transfection with the luciferase reporter, and the miR-488 overexpression and inhibiting constructs. In the assay system, a reduction in luciferase expression indicated a specific miR-448-3'-UTR interaction. It was found that the luciferase activity was markedly reduced in the high miR-448 cells, particularly in the miR-448 mimics + Rab2B-WT group $(\mathrm{P}<0.01)$. By contrast, mutation of the predicted binding sites in the Rab2B-3'-UTR eliminated the reduction of luciferase reporter activity (Fig. 3C). When combined with the results of the bioinformatics analysis, it was confirmed that miR-448 contributed to the control of the expression of Rab2B.
Overexpression of miR-448 downregulates Rab2B levels in PANC-1 cells. In PANC-1 cells transfected with miR-448 mimics, the level of miR-448 was distinctly enhanced, compared with that in the control group ( $\mathrm{P}<0.01$; Fig. $4 \mathrm{~A})$. The results of the RT-qPCR and western blot analyses also showed that the aberrant expression of miR-448 led to the significant downregulation of Rab2B at the mRNA and protein levels $(\mathrm{P}<0.05$; Fig. 4B and $\mathrm{C})$. The protein expression of Rab2B was reduced by $>50 \%$ in the miR-448 mimics group $(\mathrm{P}<0.01)$.

Overexpression of miR-448 inhibits cell proliferation and promotes apoptosis. Subsequently, the effects of the 

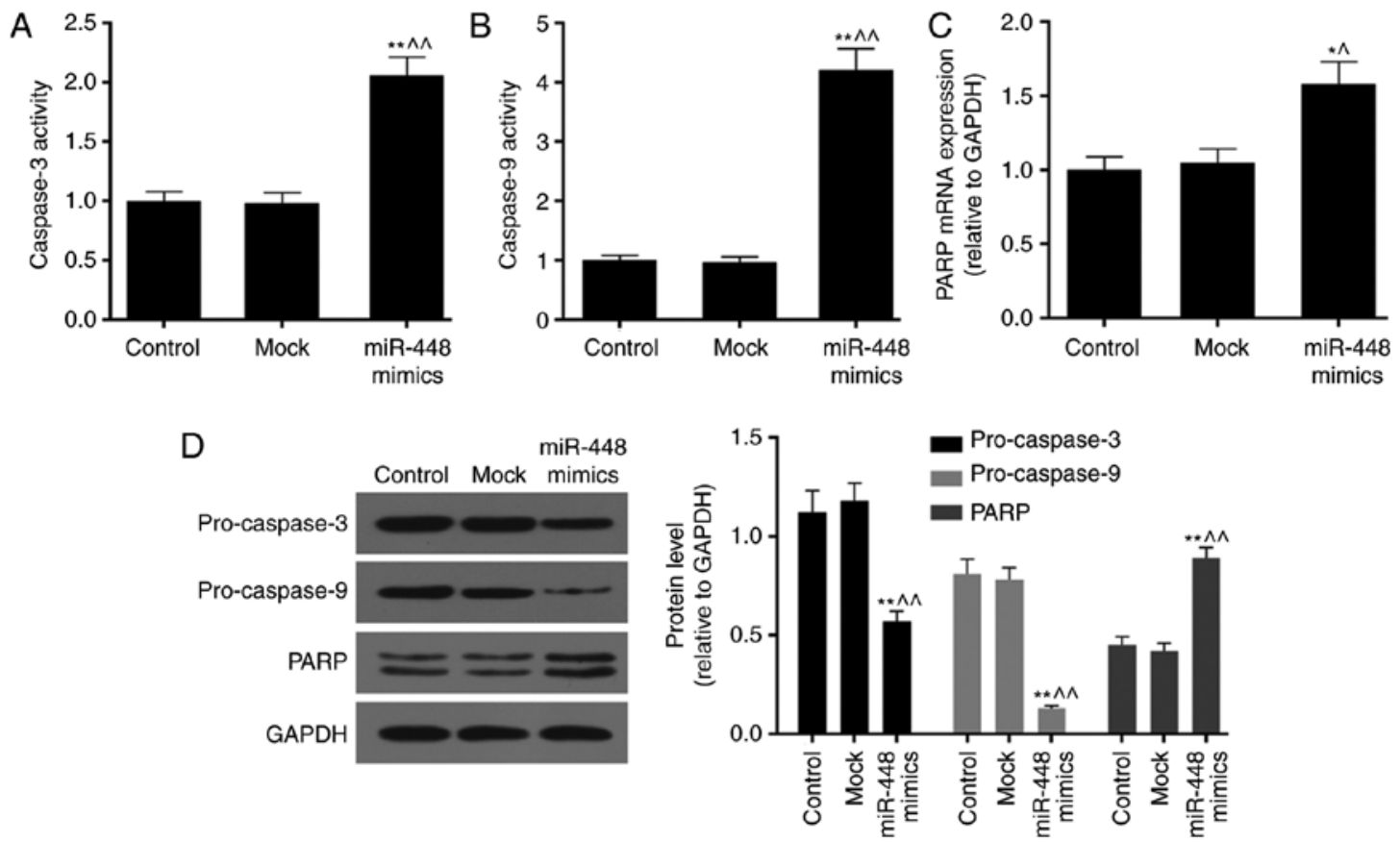

Figure 6. Overexpression of miR-448 activates caspase-3, caspase-9 and PARP. Aberrant expression of miR-448 increased the activities of (A) caspase-3 and (B) caspase-9. (C) miR-448 mimics upregulated the mRNA expression of PARP. (D) In the miR-448 mimic group, levels of pro-caspase-3 and pro-caspase-9 were decreased, whereas the protein level of PARP was increased. Data are shown as the mean \pm standard deviation of three independent experiments. ${ }^{\mathrm{P}}<0.05$, ${ }^{* *} \mathrm{P}<0.01$, vs. control; ${ }^{\wedge} \mathrm{P}<0.05,{ }^{\wedge} \mathrm{P}<0.01$, vs. mock. miR, microRNA; PARP, poly (ADP-ribose) polymerase.

overexpression of miR-448 on the biological processes of PANC-1 cells, including cell viability, cell cycle and apoptosis, were observed (Fig. 4D-F). In the miR-448 mimics group, it was found that the aberrant expression of miR-448 significantly inhibited PANC-1 cell growth and proliferation in vitro, however, it induced $\mathrm{G}_{0} / \mathrm{G}_{1}$ cell cycle arrest in comparison with the control group. A marked increase in apoptotic rate was identified under the condition of a high level of miR-448 $(\mathrm{P}<0.01)$. The above results revealed the importance of miR-448 in the development of pancreatic cancer.

Overexpression of miR-448 decreases the expression of cyclin D1 but upregulates the expression of $p 21$ and p27. The effects of aberrant miR-448 levels on the expression of cell cycle proteins cyclin D1, p21 and p27 were detected by western blot analysis (Fig. 5A). In the miR-448 overexpressing cells, the protein level of cyclin D1 was reduced by $>50 \%$, compared with that in the control $(\mathrm{P}<0.01)$. By contrast, the expression of p21 and p27 were significantly upregulated by miR-448 at the protein level $(\mathrm{P}<0.01)$.

Overexpression of miR-448 suppresses the activation of Akt, Mammalian target of rapamycin (mTOR) and S6K1. The phosphorylation levels of Akt, mTOR and S6K1 were determined using western blot analysis (Fig. 5B-D). The results showed an apparent negative correlation between a high level of miR-448 and the Akt/mTOR signaling pathway. In the presence of miR-448, the protein levels of phosphorylated Akt, mTOR and S6K1 were observed to be reduced by $>50 \%$, compared with those in the control. These results indicated inactivation of the Akt/mTOR pathway by miR-448 $(\mathrm{P}<0.01)$.
Overexpression of miR-448 increases the activities of caspase-3 and caspase-9 and upregulates the expression of poly(ADP-ribose) polymerase (PARP). The results of spectrophotometric methods showed significant increases in the activities of caspase- 3 and caspase- 9 in the miR-448 mimics group $(\mathrm{P}<0.01$; Fig. $6 \mathrm{~A}$ and $\mathrm{B})$. The protein levels of pro-caspase-3 and pro-caspase- 9 were found to be significantly decreased in the presence of a high level of miR-448 (P<0.01; Fig. 6D). miR-448 overexpression also increased the expression of PARP at the mRNA and protein levels $(\mathrm{P}<0.01$; Fig. $6 \mathrm{C}$ and $\mathrm{D})$. A model summarizing the results of the present study is shown in Fig. 7.

\section{Discussion}

As a key regulator of gene expression, miRNAs are reported to control numerous physiological and pathophysiological events in malignant tumors. A number of miRNAs have been identified to contribute to the regulation of cancer cell proliferation, apoptosis, immune evasion and metastasis (16-18). Multiple molecular target genes of miRNAs have been identified. Advances in specific inhibitors and mimics, which can be locally or systemically transferred to the organs, enable the development of miRNAs to be treated as novel therapeutic targets (19). Generally, a single miRNA is able to regulate a number of protein-coding genes. As miRNAs can identify certain 'seed sequences' in sections of genes, any gene containing a sequence complementary to the seed region is potentially regulated by the respective miRNAs (20). The results of the present study revealed a significant role of miR-448 in inhibiting the development of pancreatic cancer by regulating the expression of Rab2B in vitro. Silencing of 


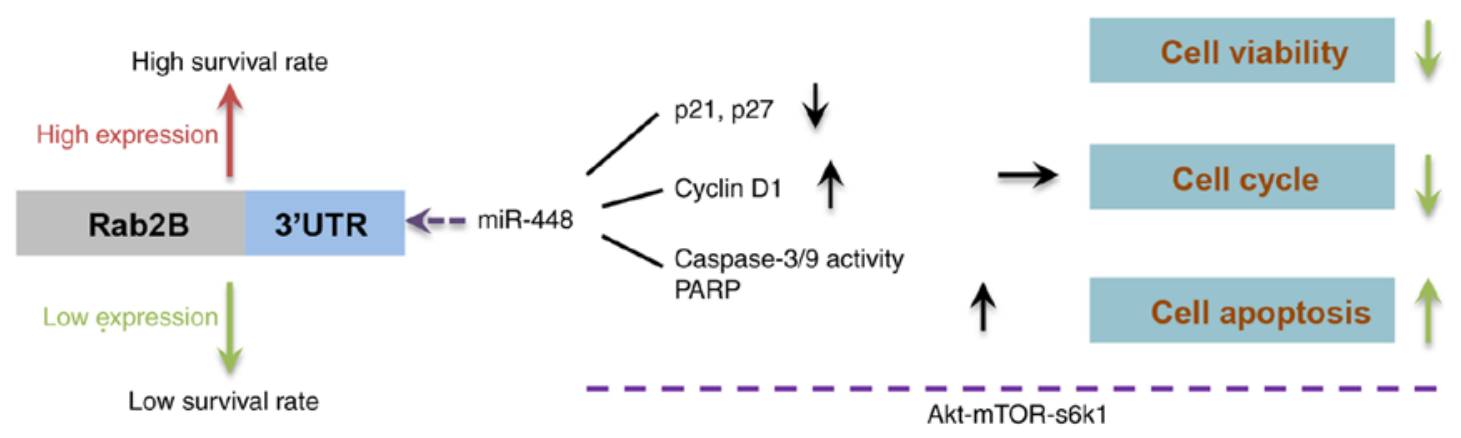

Figure 7. Summarized model of the findings of the present study. A high expression of Rab2B was associated with poor prognosis of patients with pancreatic cancer. The overexpression of miR-448 modulated the expression of p21, p27, cyclin D1, caspase-3/-9 and PARP, and induced the cell cycle arrest and apoptosis of pancreatic cancer cells. miR, microRNA; UTR, untranslated region; PARP, poly(ADP-ribose) polymerase; mTOR, Mammalian target of rapamycin.

the expression of miR-448 and a negative correlation between miR-448 and Rab2B were identified in pancreatic cancer tissues and cell lines.

Through interacting with its specific effector molecules, $\mathrm{Rab}$ is generally accepted as a key regulator of intracellular membrane trafficking. However, to date, due to the large number of Rab isoforms in mammals, specific effector molecules for the majority of mammalian Rabs have not been elucidated, and the Rab binding specificity of the previously characterized $\mathrm{Rab}$ effectors remains unclear (11). In silico analysis confirmed that Rab2B was one of 90 reliable target proteins of miR-448. At the cellular level, miR-448 mimics resulted in the apparent inhibition of cell growth and proliferation, led to a significant increase in apoptotic rate and downregulated the expression of Rab2B. These results extend those of previous reports on the role of miR-448 in pancreatic cancer and provide novel insight into how the expression of $\mathrm{Rab} 2 \mathrm{~B}$ can be regulated at the post-transcriptional level. As aberrant protein levels of Rab have been shown to be involved in certain malignant tumors, the present study provides scope for a wide range of cancer investigations $(21,22)$.

The cell cycle is divided into the $\mathrm{G}_{1}$ phase (dnapresynthetic phase), $S$ phase (synthesis phase), $\mathrm{G}_{2}$ phase (post-synthesis phase) and $M$ phase (mitotic phase). The irreversibility of these events determines the uni-polarity of the cell cycle. The cell cycle is regulated by multiple factors, including the cyclin, maturation promoting factor, growth factor, colyone and cell division cycle genes. Once the process lacks the support of the internal or external signals, cells are unable to transfer into the next phase, which may result in cycle arrest $(23,24)$. The present study applied FCM to investigate the effect of miR-448 mimics on cell cycle in PANC-1 cells. Based on the results, no significant difference in cell cycle distribution was observed between the control and mock groups. In the miR-448 mimic group, the aberrant expression of miR-448 increased the cell rate in the $G_{0} / G_{1}$ phase, but decreased the rates in the $S$ phase and $\mathrm{G}_{2} / \mathrm{M}$ phase, suggesting that miR-448 was likely to inhibit the $G_{0} / G_{1}$ cell cycle phase, and inhibit the proliferation of PANC-1 cells.

Akt is a junction of diverse cell signaling pathways. p-Akt, the activated form of $\mathrm{Akt}$, is important in enhancing cell growth and proliferation, promoting cell cycle development and suppressing apoptosis. The activation of Akt is involved in tumorigenesis and cancer metastasis (25-27). mTOR is a untypical serine/threonine protein kinase, and has attracted much attention due to its close association with tumorigenesis and the development of numerous types of cancer. S6K1, one of the downstream target proteins of mTOR, is a multifunctional kinase. When the extracellular environment changes, mTOR is involved in the regulation of cell growth, differentiation, proliferation and protein synthesis through phosphorylating S6K1. Previous studies on S6K1 have mainly focused on its role in tumorigenesis. It is hypothesized that activated S6K1 can phosphorylate substrates, inhibit apoptosis, and promote development of the cell cycle, and is also involved in vascularization $(23,28,39)$. In the present study, a significant negative correlation between a high level of miR-448 and the Akt/mTOR signal pathway was observed by western blot analysis. Upregulating the expression of miR-448 substantially suppressed the phosphorylation of $\mathrm{Ak}$, which inhibited the activation of its downstream targets, including mTOR and S6K1. Suppression of the Akt/mTOR/S6K1 pathway is likely to be closely associated with the promotion of apoptosis in pancreatic cancer through mediating associated gene expression.

The results of the present study suggested that the overexpression of miR-488 affected the expression of a series of apoptosis-related genes. Caspase-3 belongs to the CED-3 subfamily. Caspase- 3 is an inactive zymogen in the cytoplasm under a normal state, but it can be activated by caspase- 9 in apoptosis. As a key enzyme in the apoptotic process, caspase-3, which was alternatively named as apoptotic effector, is pivotal to the mitochondrial apoptosis pathway, and is also one of the important molecular mechanisms underlying tumor cell apoptosis. The activation of caspase- 3 can split corresponding substrates in the cell nucleus and cytoplasm, promote the fragmentation of DNA and finally kill cells (30). PARP, a type of modification enzyme existing in the majority of eukaryocytes, is one of the substrates of caspase- 3 and is primarily located in the cell nucleus (31). It is involved in the post-translational modification of numerous proteins and enzymes in the cell nucleus and is important in DNA damage repair, DNA duplication, regulation of cell proliferation and differentiation, apoptosis and tumorigenesis $(32,33)$. Evidence shows that the aberrant activation of PARP can trigger apoptotic signals, and induce mitochondria to release apoptosis-inducing factor, resulting in DNA cleavage and apoptosis (34-36). In pancreatic cancer cells, significantly elevated activities of caspase- 3 and 
caspase-9 were detected as a result of the aberrant expression of miR-448. The results of western blot analysis also indicated substantial reductions in the levels of pro-caspase- 3 and pro-caspase-9 and increased expression of PARP in the miR-448 mimics group. These changes suggested that miR-448 induced the apoptosis of pancreatic cancer cells.

In conclusion, miR-448 may directly target Rab2B. Aberrant levels of miR-448 in PANC-1 cells downregulated the expression of Rab2B, however, it significantly decreased cell proliferation and promoted the apoptosis of cancer cells. miR-448 mimics resulted in $G_{0} / G_{1}$ cell cycle arrest and affected the expression of cell cycle regulators (cyclin D1, p21 and p27), leading to silencing of the Akt/mTOR signal pathway, the activation of caspase-3, caspase-9 and PARP, and the induction of apoptosis. These results suggested that miR-448 was a negative regulator of Rab2B, suppressing the development of pancreatic cancer via the apoptotic pathway.

\section{Acknowledgements}

Not applicable.

\section{Funding}

The present study was supported by the National Natural Science Foundation of China (grant nos. NSFC-81572307 and NSFC-81773096), the Major Project of Medical and Health Technology Development Program in Zhejiang Province (grant no. WKJ-ZJ-1702) and the Science and Technology Major Project of Zhejiang Province (grant no. 2014C13G2010059).

\section{Availability of data and materials}

All data generated and/or analyzed during this study are included in this published article.

\section{Authors' contributions}

JJ wrote the main manuscript. YW and DZ performed the experiments. JJ and WW designed the study. JJ, YW and QS performed data analysis. JJ, YW and WW contributed to manuscript revisions and all authors reviewed the manuscript. All authors read and approved the manuscript and agree to be accountable for all aspects of the research in ensuring that the accuracy or integrity of any part of the work are appropriately investigated and resolved.

\section{Ethics approval and consent to participate}

All experimental protocols were approved by the Ethics Committee of The First Affiliated Hospital, School of Medicine, Zhejiang University. Patients provided signed written informed consent.

\section{Patient consent for publication}

Informed consent was obtained from all participants for the publication of their data.

\section{Competing interests}

The authors declare that they have no competing interests.

\section{References}

1. Hackeng WM, Hruban RH, Offerhaus GJ and Brosens LA: Surgical and molecular pathology of pancreatic neoplasms. Diagn Pathol 11: 47, 2016.

2. Martinez-Useros J and Garcia-Foncillas J: Can molecular biomarkers change the paradigm of pancreatic cancer prognosis? Biomed Res Int 2016: 4873089, 2016.

3. Siegel RL, Miller KD and Jemal A: Cancer statistics, 2017. CA Cancer J Clin 67: 7-30, 2017.

4. Abreu FB, Liu X and Tsongalis GJ: miRNA analysis in pancreatic cancer: The Dartmouth experience. Clin Chem Lab Med 55: 755-762, 2017.

5. Chari ST: Detecting early pancreatic cancer: Problems and prospects. Semin Oncol 34: 284-294, 2007.

6. Wang Z, Li Y, Ahmad A, Banerjee S, Azmi AS, Kong D and Sarkar FH: Pancreatic cancer: Understanding and overcoming chemoresistance. Nat Rev Gastroenterol Hepatol 8: 27-33, 2011.

7. McCann MJ, Rotjanapun K, Hesketh JE and Roy NC: Expression profiling indicating low selenium-sensitive microRNA levels linked to cell cycle and cell stress response pathways in the CaCo-2 cell line. Br J Nutr 117: 1212-1221, 2017.

8. Lizarbe MA, Calle-Espinosa J, Fernandez-Lizarbe E, Fernández-Lizarbe S, Robles MÁ, Olmo N and Turnay J: Colorectal cancer: From the genetic model to posttranscriptional regulation by noncoding RNAs. Biomed Res Int 2017: 7354260, 2017.

9. Narouie B, Ziaee SAM, Basiri A and Hashemi M: Functional polymorphism at the miR-502-binding site in the 3 ' untranslated region of the SETD8 gene increased the risk of prostate cancer in a sample of Iranian population. Gene 626: 354-357, 2017.

10. D'Angelo B, Benedetti E, Cimini A and Giordano A: MicroRNAs: A puzzling tool in cancer diagnostics and therapy. Anticancer Res 36: 5571-5575, 2016.

11. Fukuda M, Kanno E, Ishibashi $\mathrm{K}$ and Itoh T: Large scale screening for novel rab effectors reveals unexpected broad rab binding specificity. Mol Cell Proteomics 7: 1031-1042, 2008.

12. Baldini G, Hohl T, Lin HY and Lodish HF: Cloning of a rab3 isotype predominantly expressed in adipocytes. Proc Natl Acad Sci USA 89: 5049-5052, 1992.

13. Lutcke A, Jansson S, Parton RG, Chavrier P, Valencia A, Huber LA, Lehtonen E and Zerial M: Rab17, a novel small GTPase, is specific for epithelial cells and is induced during cell polarization. J Cell Biol 121: 553-564, 1993.

14. Tisdale EJ, Bourne JR, Khosravi-Far R, Der CJ and Balch WE: GTP-binding mutants of rab1 and rab2 are potent inhibitors of vesicular transport from the endoplasmic reticulum to the golgi complex. J Cell Biol 119: 749-761, 1992.

15. Culine S, Honore N, Closson V, Droz JP, Extra JM, Marty M, Tavitian A and Olofsson B: A small GTP-binding protein is frequently overexpressed in peripheral blood mononuclear cells from patients with solid tumours. Eur J Cancer 30A: 670-674, 1994.

16. Alma DC, Gerardo CM, Abraham PT, Romo RV, Reyes FIP, López-Urrutia E and Pérez-Plasencia C: Micro-RNAs as potential predictors of response to breast cancer systemic therapy: Future clinical implications. Int J Mol Sci 18: E1128, 2017.

17. Mandujano-Tinoco EA, Garcia-Venzor A, Munoz-Galindo L, Lizarraga-Sanchez F, Favela-Orozco A, Chavez-Gutierrez E, Krötzsch E, Salgado RM, Melendez-Zajgla J and Maldonado V: miRNA expression profile in multicellular breast cancer spheroids. Biochim Biophy Acta 1864: 1642-1655, 2017.

18. Eichmuller SB, Osen W, Mandelboim O and Seliger B: Immune modulatory microRNAs involved in tumor attack and tumor immune escape. J Natl Cancer Inst 109: 2017.

19. van Rooij E and Olson EN: MicroRNA therapeutics for cardiovascular disease: Opportunities and obstacles. Nat Rev Drug Discov 11: 860-872, 2012.

20. Kyrychenko S, Kyrychenko V, Badr MA, Ikeda Y, Sadoshima J and Shirokova N: Pivotal role of miR-448 in the development of ROS-induced cardiomyopathy. Cardiovasc Res 108: 324-334, 2015. 
21. Qin X, Wang J, Wang X, Liu F, Jiang B and Zhang Y: Targeting rabs as a novel therapeutic strategy for cancer therapy. Drug Discov Today 22: 1139-1147, 2017.

22. Dong Q, Fu L, Zhao Y, Du Y, Li Q, Qiu X and Wang E: Rablla promotes proliferation and invasion through regulation of YAP in non-small cell lung cancer. Oncotarget 8: 27800-27811, 2017.

23. Gu L, Gao J, Li Q, Zhu YP, Jia CS, Fu RY, Chen Y, Liao QK and Ma Z: Rapamycin reverses NPM-ALK-induced glucocorticoid resistance in lymphoid tumor cells by inhibiting mTOR signaling pathway, enhancing $\mathrm{G}_{1}$ cell cycle arrest and apoptosis. Leukemia 22: 2091-2096, 2008.

24. Shankland SJ, Hugo C, Coats SR, Nangaku M, Pichler RH, Gordon KL, Pippin J, Roberts JM, Couser WG and Johnson RJ: Changes in cell-cycle protein expression during experimental mesangial proliferative glomerulonephritis. Kidney Int 50: 1230-1239, 1996.

25. Zhang D and Brodt P: Type 1 insulin-like growth factor regulates MT1-MMP synthesis and tumor invasion via PI 3-kinase/Akt signaling. Oncogene 22: 974-982, 2003.

26. Roy HK, Olusola BF, Clemens DL, Karolski WJ, Ratashak A, Lynch HT and Smyrk TC: AKT proto-oncogene overexpression is an early event during sporadic colon carcinogenesis. Carcinogenesis 23: 201-205, 2002.

27. Cinti C, Vindigni C, Zamparelli A, La Sala D, Epistolato MC, Marrelli D, Cevenini G and Tosi P: Activated Akt as an indicator of prognosis in gastric cancer. Virchows Arch 453: 449-455, 2008.

28. Li JC, Zhu HY, Chen TX, Zou LY, Wang XY, Zhao HC and Xu J: Roles of mTOR and p-mTOR in gastrointestinal stromal tumors. Asian Pac J Cancer Prev 14: 5925-5928, 2013.

29. Pastor MD, Garcia-Yebenes I, Fradejas N, Pérez-Ortiz JM, Mora-Lee S, Tranque P, Moro MA, Pende M and Calvo S: mTOR/S6 kinase pathway contributes to astrocyte survival during ischemia. J Biol Chem 284: 22067-22078, 2009.
30. Colell A, Ricci JE, Tait S, Milasta S, Maurer U, Bouchier-Hayes L, Fitzgerald P, Guio-Carrion A, Waterhouse NJ, Li CW, et al: GAPDH and autophagy preserve survival after apoptotic cytochrome $c$ release in the absence of caspase activation. Cell 129: 983-997, 2007.

31. Schreiber V, Amé JC, Dollé P, Schultz I, Rinaldi B, Fraulob V, Ménissier-de Murcia J and de Murcia G: Poly(ADP-ribose) polymerase-2 (PARP-2) is required for efficient base excision DNA repair in association with PARP-1 and XRCC1. J Biol Chem 277: 23028-23036, 2002.

32. Haince JF, Rouleau M, Hendzel MJ, Masson JY and Poirier GG: Targeting poly(ADP-ribosyl)ation: A promising approach in cancer therapy. Trends Mol Med 11: 456-463, 2005.

33. Tormanen-Napankangas U, Soini Y, Kahlos K, Kinnula V and Paakko P: Expression of caspases-3, -6 and -8 and their relation to apoptosis in non-small cell lung carcinoma. Int $\mathrm{J}$ Cancer 93: 192-198, 2001

34. Cho BB and Toledo-Pereyra LH: Caspase-independent programmed cell death following ischemic stroke. J Invest Surg 21: 141-147, 2008.

35. Moroni F: Poly(ADP-ribose)polymerase 1 (PARP-1) and postischemic brain damage. Curr Opin Pharmacol 8: 96-103, 2008.

36. van Wijk SJ and Hageman GJ: Poly(ADP-ribose) polymerase-1 mediated caspase-independent cell death after ischemia/reperfusion. Free Radic Biol Med 39: 81-90, 2005.

This work is licensed under a Creative Commons Attribution-NonCommercial-NoDerivatives 4.0 International (CC BY-NC-ND 4.0) License. 